
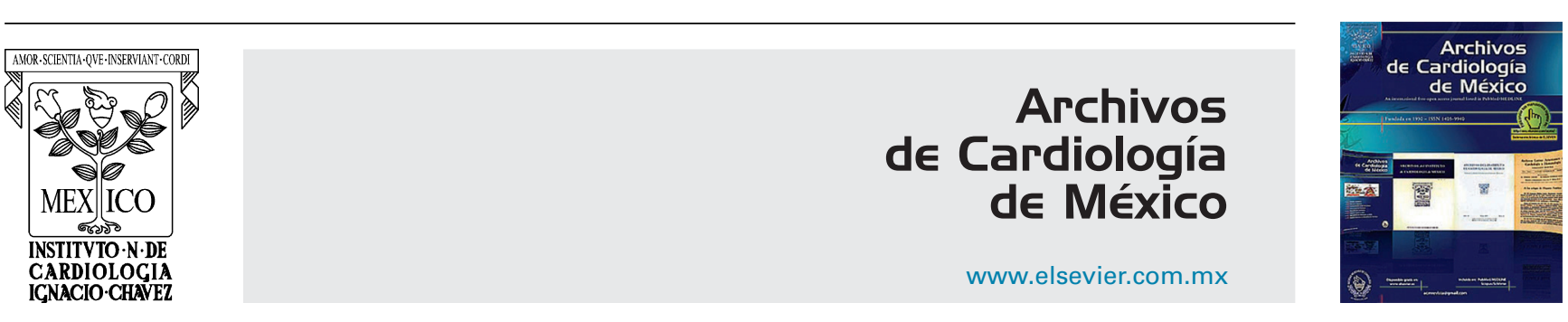

INVESTIGACIÓN BÁSICA

\title{
Anomalía de Ebstein «like» en doble entrada ventricular. Una rara asociación
}

\author{
Luis Muñoz Castellanos ${ }^{\mathrm{a}, *}$ y Magdalena Kuri Nivon ${ }^{\mathrm{b}}$
}

\author{
a Departamento de Embriología, Instituto Nacional de Cardiología Ignacio Chávez, Ciudad de México, México \\ ${ }^{\mathrm{b}}$ Escuela Superior de Medicina Instituto Politécnico Nacional, Ciudad de México, México
}

Recibido el 18 de diciembre de 2015; aceptado el 25 de noviembre de 2016

\author{
PALABRAS CLAVE \\ Cardiopatía congénita \\ compleja; \\ Doble entrada \\ ventricular con \\ Ebstein «like»; \\ Conexión \\ auriculoventricular \\ univentricular; \\ Corazón \\ univentricular; \\ México
}

\begin{abstract}
Resumen
Introducción y objetivos: La asociación de corazones univentriculares con doble entrada y anomalía de Ebstein «like» de la válvula auriculoventricular común es extremadamente rara. Se describen 2 corazones con esta asociación.

Métodos: Se utilizó el sistema secuencial segmentario que determina el situs auricular, los tipos y modos de conexión auriculoventricular y ventriculoarterial y las anomalías agregadas.

Resultados: Ambos corazones presentaron situs solitus auricular, defecto septal auriculoventricular con foramen primum y doble entrada y doble salida con grandes arterias normalmente relacionadas en un ventrículo único. En el primero las 4 valvas auriculoventriculares estuvieron adosadas a la pared ventricular desde la unión auriculoventricular hasta el ápex con auricularización de la entrada y de la porción trabecular y presentó estenosis infundibular y valvular. En el segundo la porción proximal de la válvula auriculoventricular estuvo adosada a la pared ventricular con auricularización pequeña; en su porción distal mostró displasia con nodulaciones fibromixoides y cuerdas tendinosas pequeñas y gruesas y la arteria pulmonar estuvo dilatada. Conclusiones: Ambos corazones forman parte de la conexión auriculoventricular univentricular del sistema secuencial segmentario, cuya precisión diagnóstica muestra su eficacia. Las asociaciones de defectos congénitos en un solo corazón muestran el infinito espectro de las mismas, lo que expande nuestro conocimiento de la cardiología pediátrica.

(C) 2016 Instituto Nacional de Cardiología Ignacio Chávez. Publicado por Masson Doyma México S.A. Este es un artículo Open Access bajo la licencia CC BY-NC-ND (http://creativecommons. org/licenses/by-nc-nd/4.0/).
\end{abstract}

\footnotetext{
* Autor para correspondencia. Departamento de Embriología, Instituto Nacional de Cardiología Ignacio Chávez, Juan Badiano, núm. 1, Colonia Sección 16, Delegación Tlalpan México, D.F. 14080. Teléfono: 5573-2911; ext. 1257.

Correos electrónicos: munozembriologia1@gmail.com, munoz-embriologia@hotmail.com (L. Muñoz Castellanos).
} 


\section{KEYWORDS}

Congenital heart disease;

Double inlet ventricle with Ebstein's "like" anomaly; Atrioventricular univentricular connection; Univentricular heart; Mexico

\section{Introducción}

La anomalía de Ebstein originalmente fue descrita como una malformación de la válvula tricúspide y del ventrículo derecho en un corazón con conexión auriculoventricular concordante. Se caracteriza por adosamiento de las valvas septal y posterior a la pared ventricular con redundancia de la valva anterior generalmente no adosada; las valvas presentan nodulaciones fibromixoides, cuerdas tendinosas cortas, engrosadas o ausentes ${ }^{1-3}$. El desplazamiento valvar es variable y produce un espectro de grados de auricularización del ventrículo derecho con reducción de la porción funcional de esta cámara (fig. 1). En el presente trabajo se describen 2 corazones con conexión auriculoventricular univentricular del tipo doble entrada a un ventrículo único con anomalía de Ebstein «like» de la válvula auriculoventricular común, asociación extremadamente rara de la que no se encontraron referencias en la literatura. La descripción de estos especímenes con el sistema secuencial segmentario demuestra la gran flexibilidad de este método diagnóstico ${ }^{4}$. Se analizan las diferencias de la anomalía de Ebstein cuando se presenta en distintas conexiones auriculoventriculares: concordante, discordante y doble entrada ventricular.

La doble entrada ventricular es un tipo de conexión auriculoventricular univentricular en la que ambas aurículas se abren a un solo ventrículo y presenta 3 modalidades: en ventrículo izquierdo ${ }^{5}$, en ventrículo derecho ${ }^{6}$ y en ventrículo único a través de 2 válvulas auriculoventriculares o de una válvula auriculoventricular común ${ }^{7,8}$ (figs. 2 y 3 ).

Se define al ventrículo único cuando existe en la masa ventricular una sola cámara que en su porción de entrada presenta la conexión auriculoventricular y en su porción de

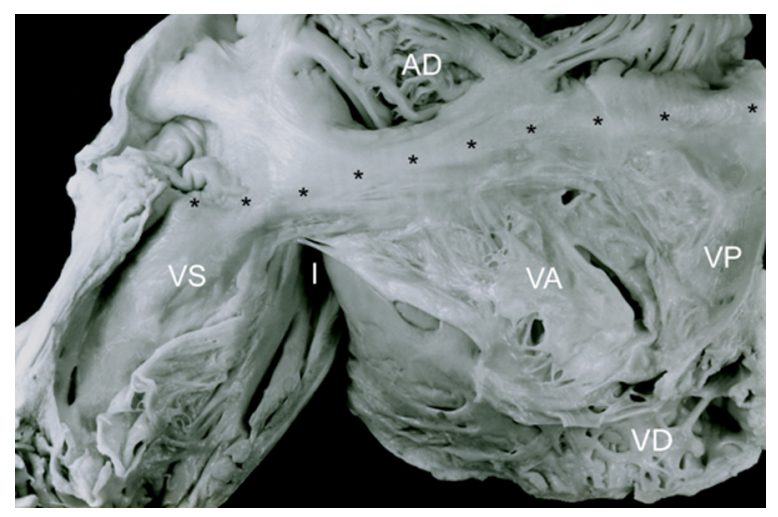

Figura 1 Vista interna de las cámaras derechas de un corazón con concordancia auriculoventricular y anomalía de Ebstein de la válvula tricúspide. El anillo fibroso está situado en la unión auriculoventricular (asteriscos). Obsérvese el adosamiento de las valvas septal y posterior a las paredes ventriculares, la valva anterior es redundante. AD: aurícula derecha; I: infundíbulo; VA: válvula anterior; VD: ventrículo derecho; VP: válvula posterior; VS: válvula septal.

salida emergen las grandes arterias o una sola arteria, el tronco aórtico solitario ${ }^{9,10}$ (fig. 4).

\section{Material y método}

Se describieron morfológicamente 2 corazones univentriculares con doble entrada y anomalía de Ebstein de la válvula auriculoventricular común que forman parte de la colección 

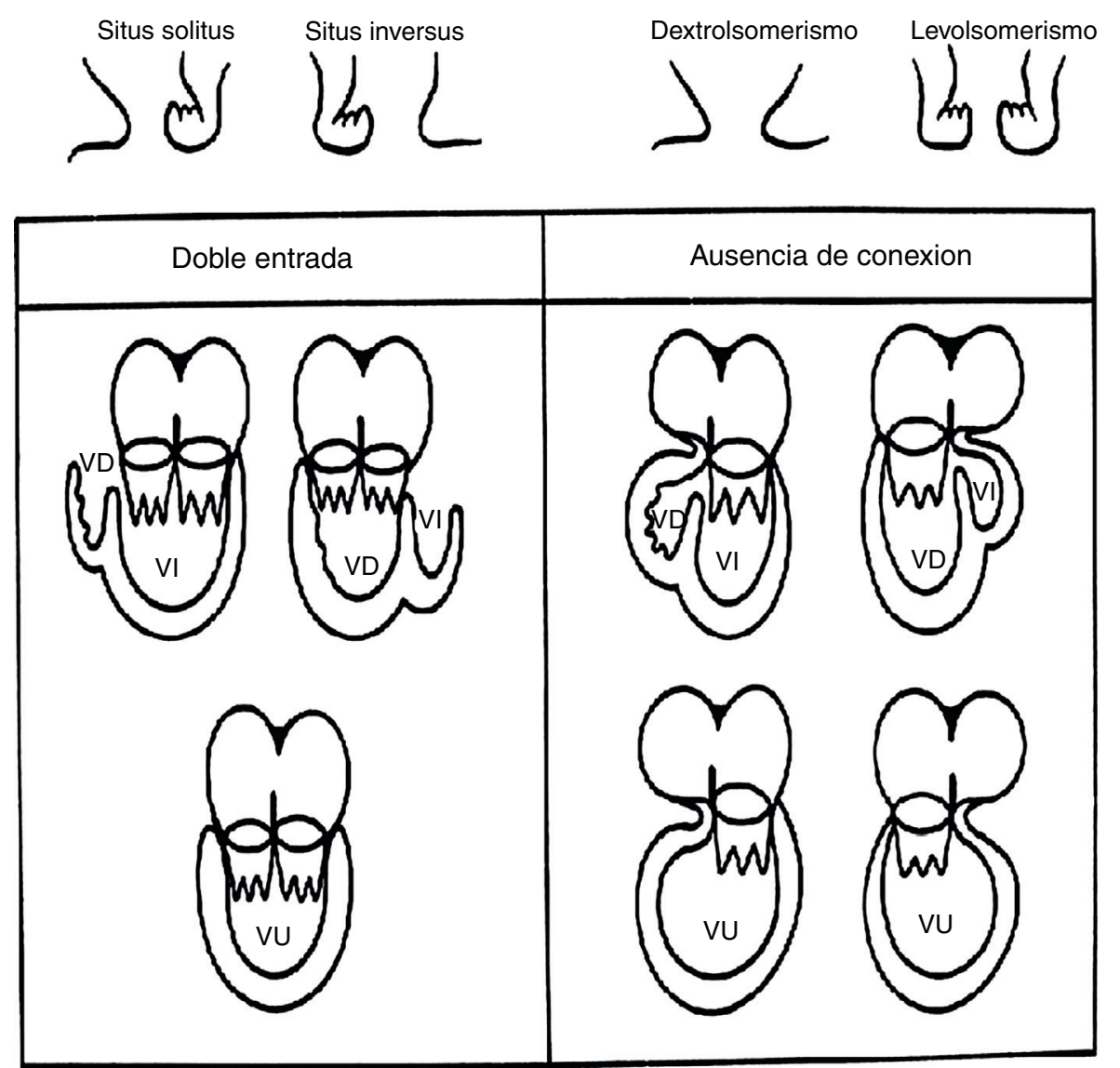

Figura 2 Esquemas que muestran el concepto de conexión auriculoventricular univentricular que comprende 2 tipos de conexión: doble entrada y ausencia de conexión en sus respectivas modalidades. VD: ventrículo derecho; VI: ventrículo izquierdo; VU: ventrículo único.

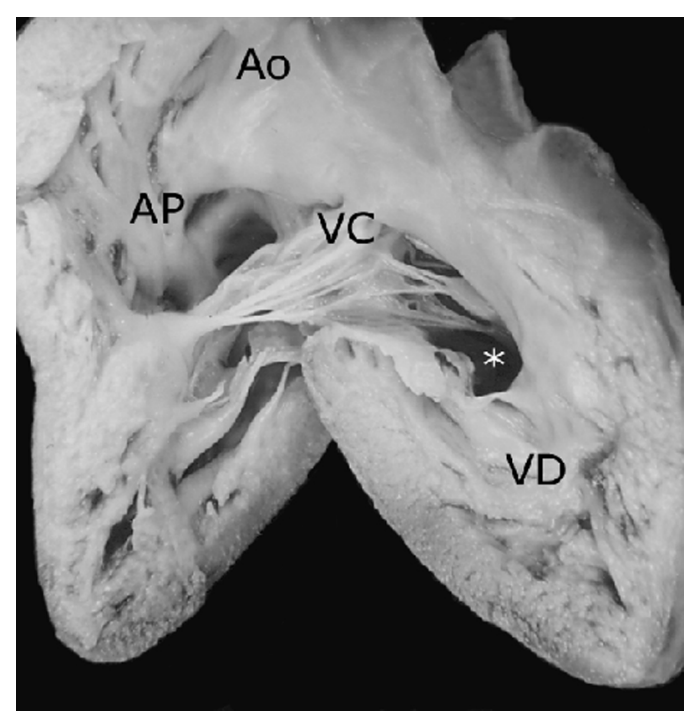

Figura 3 Vista interna del ventrículo derecho con doble entrada a esta cámara. Obsérvese la válvula auriculoventricular común conectada en más del $75 \%$ con el ventrículo derecho. Ao: aorta; AP: arteria pulmonar; VC: válvula común; VD: ventrículo derecho. Asterisco: comunicación interventricular.

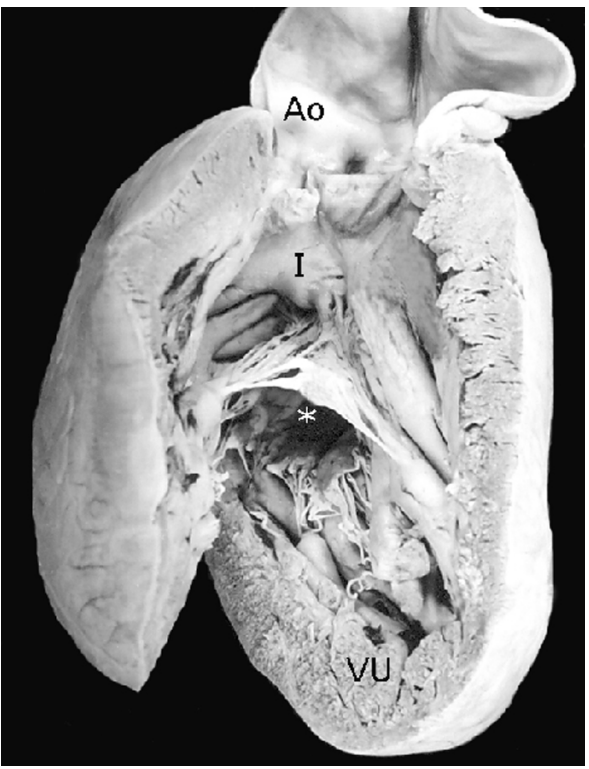

Figura 4 Vista interna de un corazón univentricular indeterminado. Obsérvese la porción de entrada representada por la válvula auriculoventricular común (asterisco) y por encima de esta la única vía de salida o infundíbulo del que emerge un tronco aórtico solitario. Ao: aorta; I: infundíbulo; VU: ventrículo único. 


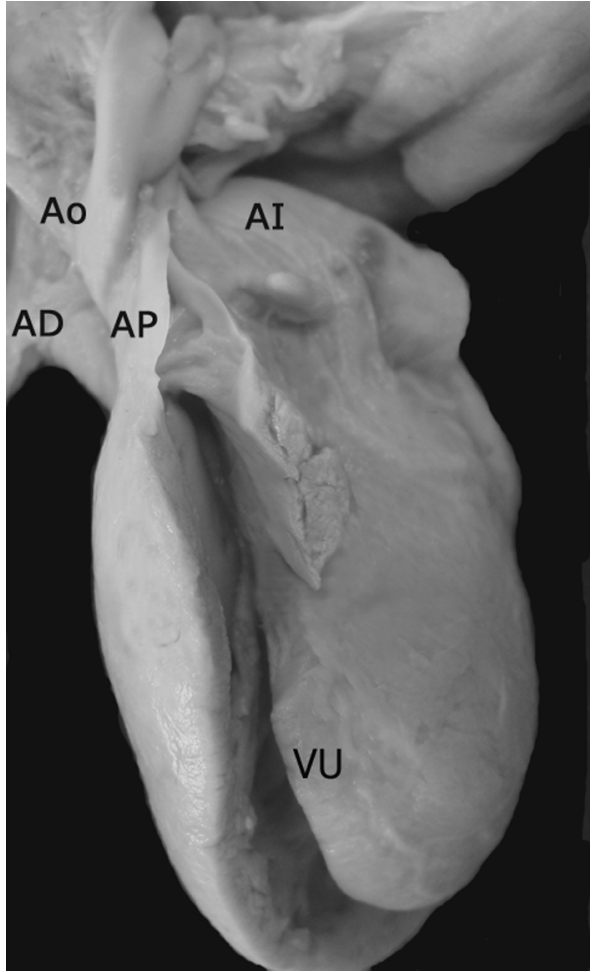

Figura 5 Vista externa anterior de un corazón en situs solitus auricular con doble entrada en ventrículo único y anomalía de Ebstein de la válvula auriculoventricular común. Las grandes arterias están normalmente relacionadas y emergen del ventrículo único. Obsérvese la porción funcional del ventrículo único ubicada en la zona trabecular anterior y en las zonas subinfundibular e infundibular. AD: aurícula derecha; Ao: aorta; Al: aurícula izquierda; AP: arteria pulmonar; VU: ventrículo único.

patológica del Instituto Nacional de Cardiología Ignacio Chávez. Se utilizó como método descriptivo morfológico el sistema secuencial segmentario empleado en el diagnóstico de las cardiopatías congénitas ${ }^{4}$. Se determinaron el situs auricular, los tipos y modos de conexión auriculoventricular y ventriculoarterial, los defectos septales y las anomalías asociadas; en especial se describieron las características anatómicas de la válvula auriculoventricular. Se determinó el grado de adosamiento y desplazamiento valvares con el método de Becker et al. ${ }^{1}$. Para establecer las porciones auricularizada y ventricular funcional; se compara la anomalía de Ebstein de la válvula tricúspide con la de estos corazones univentriculares para resaltar sus rasgos comunes y sus diferencias básicas

\section{Resultados}

Los 2 corazones formaron parte de un situs solitus auricular, ambos con doble entrada a ventrículo único a través de una válvula auriculoventricular común cuyas valvas estuvieron adosadas a la pared ventricular; las grandes arterias normalmente relacionadas emergieron del ventrículo único con estenosis pulmonar infundibular y valvular (fig. 5); el adosamiento y desplazamiento valvares cubrieron toda la porción de entrada y la zona trabecular posterior hasta la región apical con una gran

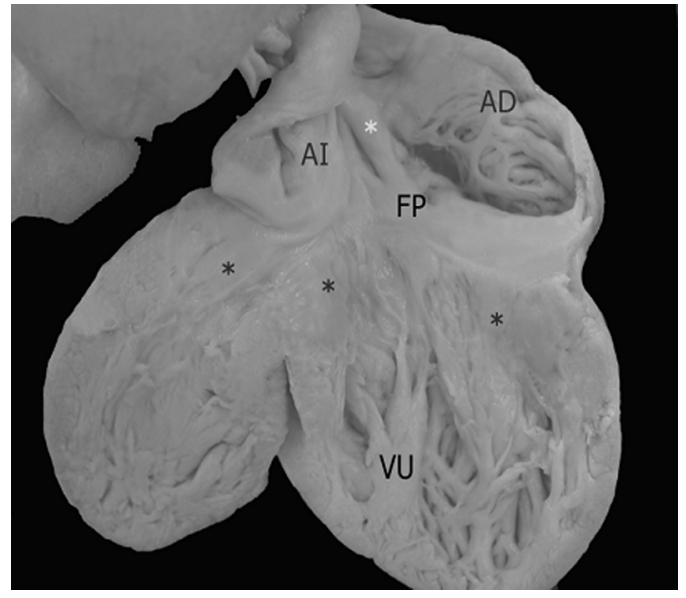

Figura 6 Vista interna posterior del corazón de la figura 5. Obsérvese las aurículas en situs solitus conectadas con el ventrículo único, las valvas de la válvula auriculoventricular adosadas a las paredes de esta cámara ventricular (3 asteriscos negros) desde la unión auriculoventricular hasta la región trabecular apical, el tabique interauricular rudimentario y un foramen primum. AD: aurícula derecha; Al: aurícula izquierda; FP: foramen primum; VU: ventrículo único.

auricularización del ventrículo único; estuvieron ausentes las cuerdas tendinosas y los músculos papilares (fig. 6); la porción funcional efectiva del ventrículo se redujo a la zona trabecular anterior y a la región infundibular (fig. 5). En el segundo corazón las grandes arterias estuvieron también normalmente relacionadas pero con dilatación y ambas emergieron del ventrículo

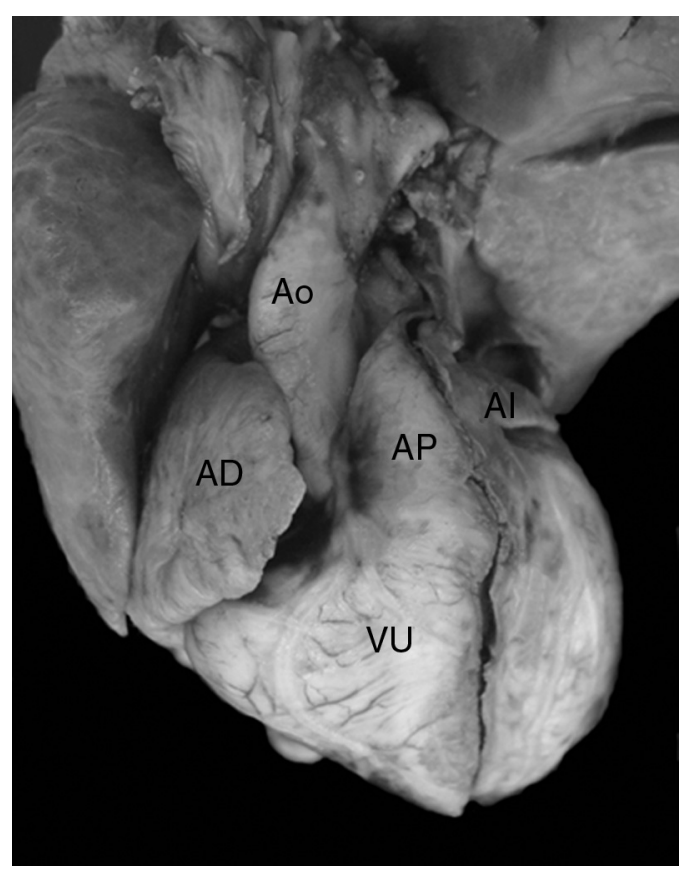

Figura 7 Vista externa frontal de un corazón en situs solitus auricular con ventrículo único que muestra la emergencia de las grandes arterias normalmente relacionadas a partir de esta cámara. AD: aurícula derecha; Al: aurícula izquierda; FP: foramen primum; VU: ventrículo único. 
Tabla 1 Semejanzas y diferencias entre los corazones con ventrículo único, con doble entrada y doble salida

\begin{tabular}{lll}
\hline Morfología & Corazón $\mathrm{n} .^{\circ} 1$ & ${\text { Corazón } \mathrm{n} .{ }^{\circ} 2}$ \\
\hline Válvula A-V común & + & + \\
Adosamiento valvar & Máximo & Mínimo \\
Auricularización & Máximo & Mínimo \\
Displasia & + & + \\
Doble salida & + & + \\
Grandes arterias normalmente relacionadas & + & + \\
& Estenosis pulmonar & Dilatación pulmonar \\
\hline
\end{tabular}

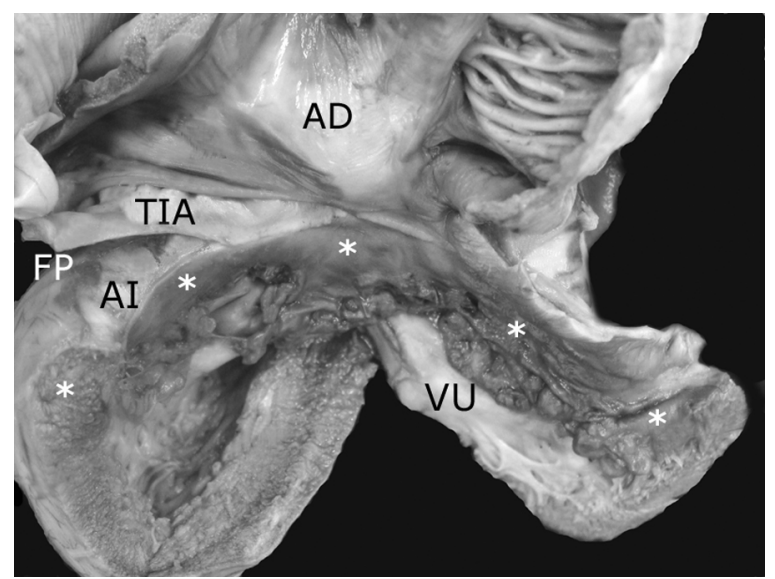

Figura 8 Vista interna posterior del corazón de la figura 7 que muestra las aurículas en situs solitus conectadas con el ventrículo único a través de una válvula auriculoventricular común. Obsérvese el adosamiento valvar a la pared del ventrículo único en su porción proximal (asteriscos) y la presencia de nodulaciones fibromixoides en su porción distal libre. La porción adosada corresponde a la zona auricularizada y el resto a la porción funcional del ventrículo único. AD: aurícula derecha; Al: aurícula izquierda; FP: foramen primum; TIA: tabique interauricular; VU: ventrículo único..

único (fig. 7). El adosamiento y desplazamiento valvares estuvieron reducidos a la porción proximal de la válvula por lo que la auricularización fue menor y mayor la porción funcional del ventrículo único (fig. 8). Ambos corazones tuvieron un tabique interauricular pequeño con foramen primum (tabla 1).

\section{Discusión}

Al comparar los corazones descritos en este trabajo con los que presentan anomalía de Ebstein de la válvula tricúspide con conexiones auriculoventriculares concordante y discordante se observan diferencias entre ellos. En la anomalía de Ebstein con conexión auriculoventricular concordante el adosamiento se presenta en forma de un espectro de grados de severidad con nodulaciones fibromixoides en las valvas liberadas, cuerdas tendinosas cortas y engrosadas y músculos papilares de estructura fibrosa o ausentes (fig. 1). En la anomalía de Ebstein con discordancia auriculoventricular el adosamiento de la valva septal es de menor extensión y afecta solamente a esta valva (fig. 9) y cuando esta malformación valvular se asocia a corazones con conexiones

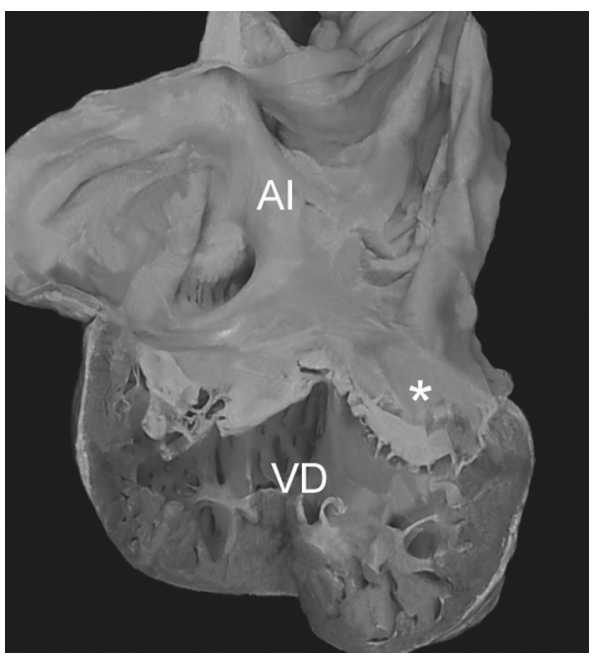

Figura 9 Vista interna de las cámaras cardíacas de un corazón con discordancia auriculoventricular y anomalía de Ebstein de la válvula tricúspide. Obsérvese la conexión de la aurícula izquierda con el ventrículo derecho, el adosamiento de la valva septal de la válvula tricúspide (asterisco). Al: aurícula izquierda; VD: ventrículo derecho.

auriculoventriculares univentriculares, el adosamiento valvar puede ser extenso o solo en las porciones proximales (figs. 6 y 8).

La anomalía de Ebstein de la válvula tricúspide se presenta con más frecuencia en corazones con conexión auriculoventricular concordante, es menos frecuente en la conexión auriculoventricular discordante y excepcionalmente rara en corazones con conexión auriculoventricular univentricular ${ }^{8}$ como los que se describen en este trabajo. De lo anterior se desprende que el patrón morfológico de la anomalía de Ebstein es diferente en cada tipo de conexión auriculoventricular mencionado y que la característica básica que define a la anomalía es el adosamiento del tejido valvar a la pared ventricular. Los corazones con conexión auriculoventricular univentricular que presentan una válvula auriculoventricular común tienen un foramen primum originado por un deficiente crecimiento del tabique interauricular. Las conexiones ventriculoarteriales más frecuentes en la doble entrada del ventrículo único es la doble salida de las grandes arterias con válvulas arteriales permeables con estenosis pulmonar o con atresia de esta válvula.

La anomalía de Ebstein de la válvula tricúspide difiere de su homóloga en la válvula auriculoventricular común en la doble entrada del ventrículo único; en la primera las 
valvas septal y posterior generalmente están adosadas y desplazadas apicalmente, mientras que la valva anterior es libre, redundante y en muchos casos displásica. En la válvula auriculoventricular común del corazón univentricular el patrón morfológico más frecuente es de 4 valvas, 2 anteriores y 2 posteriores, y no existe una valva septal ya que está ausente un tabique ventricular; en lo que sí se pueden comparar es en los estigmas patológicos que comparten ambas formas de la anomalía de Ebstein: el adosamiento valvar, el desplazamiento apical del tejido valvular y del orificio funcional así como la división del ventrículo derecho en una porción auricularizada y una zona funcional efectiva.

Con relación al ventrículo único ha existido una variada nomenclatura lo que ha propiciado múltiples controversias; en la mayoría de las sistematizaciones se acuñó el término de ventrículo único para denominar corazones con estructura biventricular lo que constituyó una contradicción ${ }^{11-13}$, por ello se prefirió describir morfológicamente esos corazones, por ejemplo el ventrículo único izquierdo correspondió a la doble entrada en ventrículo izquierdo, el ventrículo único de tipo derecho fue equivalente a la doble entrada en ventrículo derecho y el ventrículo único de tipo indeterminado correspondió al verdadero corazón univentricular como los especímenes de este estudio. Para determinar la naturaleza de estas malformaciones cardíacas Anderson et al. ${ }^{7,8}$ propusieron el concepto de conexión auriculoventricular univentricular en la que como su nombre indica un solo ventrículo recibe la o las conexiones auriculoventriculares y aquí se incluyó además de la doble entrada ventricular la ausencia de conexión auriculoventricular (fig. 2).

Los 2 corazones de este estudio también presentan diferencias entre sí; en el primero la zona auricularizada forma un saco de tejido valvar adosado a la pared miocárdica desde la unión auriculoventricular muy dilatada que termina en la región apical en fondo ciego desde el cual se comunica con la zona función efectiva del ventrículo derecho a través de pequeñas fenestraciones que permiten la entrada de sangre hacia esta zona reducida del ventrículo derecho funcional y de ahí hacia las vías de salida y hacia las grandes arterias. En el segundo espécimen el adosamiento fue menor con displasia de la poción libre con cuerdas tendinosas cortas; en ambos casos la válvula auriculoventricular común fue disfuncionante en vida del paciente; en ambos corazones las aurículas estuvieron dilatadas, el tabique interauricular fue pequeño con un foramen primum, estigmas que se presentan cuando existe un defecto de la tabicación auriculoventricular que en los corazones de este estudio se combinó con ventrículo.

El termino displasia denota una anomalía intrínseca del desarrollo de una estructura particular. Un ejemplo bien conocido de displasia que ocurre en el corazón es la anomalía de Ebstein de la válvula tricúspide, la cual se debe a un trastorno en el proceso de socavamiento y delaminación de la pared del ventrículo derecho que normalmente conduce a la liberación de una capa interna de músculo ventricular la cual se divide en 3 faldones que se diferencian en las 3 valvas y su aparato tensor de la válvula tricúspide, proceso que estudió Van Mierop en el corazón embrionario normal para explicar la morfogénesis de la válvula tricúspide. En la anomalía de Ebstein el proceso de socavamiento y delaminación es incompleto, no ocurre a nivel de las valvas y en ocasiones de la zona tendinosa por lo tanto no se diferencian las cuerdas tendinosas y en ocasiones severas tampoco se delamina la zona de los músculos papilares por lo tanto estos también estarán ausentes. En el primer corazón aquí descrito el adosamiento valvar formó un saco adherido a la pared miocárdica desde la unión auriculoventricular hasta el ápex, lo que se puede interpretar en base a la hipótesis de van Mierop como una ausencia de diverticulización y delaminación de los 4 faldones que constituyen el origen de la válvula auriculoventricular común cada uno con sus territorios valvular tendinoso y papilar, estructuras que quedaron adheridas a la pared miocárdica lo cual explica la ausencia de cuerdas tendinosas y músculos papilares lo que contrasta con el segundo espécimen donde la delaminación no ocurrió en la zona proximal de la válvula ${ }^{14}$. Esta sugerencia patogenética no permite afirmar que la anomalía que muestran los 2 corazones univentriculares se podrían caracterizar como tipo Ebstein «like» porque no son un retrato del Ebstein original descrito para la válvula tricúspide.

\section{Conclusiones}

La asociación de anomalía de Ebstein «like» en corazones univentriculares es muy rara; no se encontraron referencias de ella en la literatura.

La aplicación del sistema secuencial segmentario en la descripción de estos corazones con conexión auriculoventricular univentricular comprueba la eficacia diagnóstica de este método.

La flexibilidad de este sistema de diagnóstico morfológico de las cardiopatías congénitas permite describir con precisión todas las malformaciones por muy complejas que estas sean.

Los corazones aquí descritos muestran el infinito espectro de presentación de las cardiopatías congénitas.

\section{Responsabilidades éticas}

Protección de personas y animales. Los autores declaran que para esta investigación no se han realizado experimentos en seres humanos ni en animales.

Confidencialidad de los datos. Los autores declaran que en este artículo no aparecen datos de pacientes.

Derecho a la privacidad y consentimiento informado. Los autores declaran que en este artículo no aparecen datos de pacientes.

\section{Financiación}

No se recibió patrocinio de ningún tipo para llevar a cabo este artículo.

\section{Conflicto de intereses}

Los autores declaran no tener ningún conflicto de intereses.

\section{Agradecimientos}

Agradecemos el trabajo secretarial de la Sra. María del Rosario Soriano Velázquez. 


\section{Bibliografía}

1. Becker AE, Becker MJ, Edwards E. Pathologic spectrum of displasia of the tricuspid valve: Features in common with Ebstein's malformation. Arch Pathol. 1972;91:167-78.

2. Kuri M, Muñoz Castellanos L, Salinas HC. Estudio morfológico de 23 corazones con anomalía de Ebstein. Arch Inst Cardiol Mex. 1995;65:197-206.

3. Anderson KR, Zuberbuhler FJ, Anderson RH, et al. Morphologic spectrum of Ebstein's anomaly of the heart. A review. Mayo Clinic Proc. 1979;54:174-80.

4. Tynan M, Becker AE, McCartney FJ, et al. Nomenclature and classification in congenital heart disease. $\mathrm{Br}$ Heart $\mathrm{J}$. 1979;41:544-53.

5. De la Cruz MV, Miller BL. Double inlet left ventricle: Two pathological specimens with comments on the embryology and on its relations to single ventricle. Circulation. 1968;37: 249-60.

6. Muñoz-Castellanos L, de la Cruz MV, Cieslinsky A. Double inlet right ventricle with comments on embryology. $\mathrm{Br}$ Heart J. $1973 ; 35: 292-7$
7. Anderson RH, McCartney FJ, Tynan M, et al. Univentricular atrioventricular connection: The single ventricle trap unsprung. Pediatr Cardiol. 1983;4:273-80.

8. Anderson RH, Becker AE, Tynan M, et al. The univentricular atrioventricular connection: Getting to the root of a thorny problem. Am J Cardiol. 1984;54:828-82.

9. Becker AE, Anderson RH. Pathology of congenital heart disease. London: Butterworth's; 1981. p. 152-5.

10. Van Praagh R, David I, van Praagh S. A question of definition. Wath is a ventricle? The single ventricle trap. Pediatr Cardiol. 1982;2:79-84.

11. Van Praagh R, Ongley PA, Swan HJC. Anatomic type of single or common ventricle in man. Morphologic and geometric aspects of 60 necropsies cases. Am J Cardiol. 1964;13:367-86.

12. Van Praagh R, Plett JA, van Praagh S. Single ventricle. Pathology, embryology, terminology and classification. Herz. 1979;4:113-50

13. Lev M, Liberthson RR, Kirkpatrick JR, et al. Single (primitive) ventricle. Circ. 1969;39:577-91.

14. Van Mierop LH, Gessner I. Pathogenetic mechanisms in congenital cardiovascular malformations. Prog Cardiovasc Dis. 1972;15:67-85. 